\title{
Narrative review: the holy grail: update on pharmacotherapy for heart failure with preserved ejection fraction
}

\author{
Pablo Alejandro Paz ${ }^{1}$, Barbara Daniela Mantilla ${ }^{1}$, Erwin E. Argueta ${ }^{2}$, Debabrata Mukherjee ${ }^{3}$ \\ ${ }^{1}$ Department of Internal Medicine, Texas Tech University Health Sciences Center, Lubbock, TX, USA; ${ }^{2}$ Division of Cardiovascular medicine, Texas \\ Tech University Health Sciences Center, Lubbock, TX, USA; ${ }^{3}$ Division of Cardiovascular Medicine, Texas Tech University Health Sciences Center, \\ El Paso, TX, USA \\ Contributions: (I) Conception and design: PA Paz, EE Argueta; (II) Administrative support: None; (III) Provision of study materials or patients: None; \\ (IV) Collection and assembly of data: PA Paz, BD Mantilla; (V) Data analysis and interpretation: None; (VI) Manuscript writing: All authors; (VII) \\ Final approval of manuscript: All authors. \\ Correspondence to: Pablo Alejandro Paz, MD. Department of Internal Medicine, Texas Tech University Health Sciences Center, Lubbock, $36014^{\text {th }}$ St., \\ STOP 9410, Office 4B076 Lubbock, TX 79430, USA. Email: pablo.paz@ttuhsc.edu.
}

\begin{abstract}
Heart failure with preserved ejection fraction (HFpEF) is the presence of clinical signs and/ or symptoms of heart failure with a left ventricular ejection fraction (LVEF) $\geq 50 \%$. Risk factors associated with this disease include hypertension, hyperlipidemia, atrial fibrillation (AF), obesity, diabetes and coronary artery disease (CAD). Despite the multiple risk factors identified for this condition, treatment and management remain challenging and a subject of ongoing research. Since a treatment approach that alters the natural course or lowers mortality for this disease has not been found, treating co-morbidities and symptom management is essential. From the comorbidities, hypertension is identified as the main risk factor for disease development. Thus, after congestive symptom control with diuretics, blood pressure (BP) management is considered one of the most important preventive measures and also a target for treatment. Amongst antihypertensives, angiotensin receptor blockers (ARBs) and aldosterone antagonists are the therapeutic agents used that have a role in reducing hospitalizations. Implantable monitoring devices have also been shown to reduce hospitalizations in comparison to standard heart failure therapies by allowing to tailor diuretic therapy based on ongoing hemodynamic data. In this manuscript we discuss pharmacologic strategies for HFpEF patients by risk factors, including those with and without a potential role.
\end{abstract}

Keywords: Heart failure (HF); preserved ejection fraction; pharmacotherapy; comorbidities

Submitted Jun 10, 2020. Accepted for publication Jul 30, 2020.

doi: $10.21037 /$ atm-20-4602

View this article at: http://dx.doi.org/10.21037/atm-20-4602

\section{Introduction}

Heart failure (HF) has been classically defined as the inability of the heart to provide adequate organ perfusion at a rate that can satisfy the metabolic demands of the body, or the ability to do it at expense of elevated filling pressures (1). The previous definition applies to both heart failure with preserved ejection fraction (HFpEF) and heart failure with reduced ejection fraction (HFrEF). HF with preserved ejection fraction (EF), also referred to as diastolic $\mathrm{HF}$, is the presence of clinical signs or symptoms of HF, a left ventricular ejection fraction (LVEF) $\geq 50 \%$ and evidence of cardiac dysfunction as a cause of symptoms [e.g., abnormal left ventricle (LV) filling and elevated filling pressures]. Of note, the EF cut-off for HFrEF is $<40 \%$. The range $41-49 \%$ has been designated heart failure with mid-range ejection fraction (HFmrEF), though evidence supports that this group should be more accurately classified as HFrEF (2-4).

Over the last years the incidence of HF has remained stable but the prevalence has increased due to the likelihood of survival after diagnosis, and patients with HFpEF 
represent almost half of this population (5-7). Particularly, the $\mathrm{HFpEF}$ prevalence has been growing steadily at a rate of $10 \%$ per decade and the hospital admission proportion caused by HFpEF has continued to increase. Between January 2005 and December 2010 there were a total of 110,621 patients admitted with HF and were subclassified as: $50 \%$ with $\mathrm{HFrEF}, 14 \%$ with $\mathrm{HFmrEF}$ and $36 \%$ with HFpEF $(6,8)$. Data shows that patients with HFpEF are significantly older, more likely to be female, have hypertension, kidney disease, pulmonary disease and atrial fibrillation (AF) than those with HFrEF (8-10). Other risk factors include obesity, diabetes and coronary artery disease (CAD), with the increase in HFpEF prevalence being secondary to the increase in risk factors in the general population $(6,11)$. The reported mortality in patients with HFpEF varies depending on the study design, study population risks and EF cut-off used (12) varying from $4-5 \%$ yearly $(13,14)$ to $53-74 \%$ at five years $(6,15,16)$ with an increased risk of death when the comorbidity burden increases $(17,18)$.

By performing a comprehensive literature review of published articles in databases and synthesizing relevant information, we discussed the management strategies for HFpEF, with an emphasis on pharmacotherapy, based on the co-morbid disease targeted in this population.

We present the following article in accordance with the Narrative Review reporting checklist (available at http:// dx.doi.org/10.21037/atm-20-4602).

\section{Pharmacotherapy for common associated morbidities}

Although a treatment that alters the natural course or lowers mortality for HFpEF has not been found, treating the co-morbidities is essential for management. These include: obesity, hypertension, $\mathrm{AF}, \mathrm{CAD}$, diabetes, COPD, anemia, chronic kidney disease, and sleep-disordered breathing (19). Most co-morbidities share a common proinflammatory state with increased oxidative stress, impaired nitric oxide bioavailability and endothelial dysfunction contributing to HFpEF progression (20). This was described in a prospective HF long-term European registry after a one year follow-up period. Additionally, death from non-cardiovascular causes is more common in patients who have HFpEF than in those with a reduced $\mathrm{EF}$ (60\% vs. $35 \%$ respectively), illustrating the importance of addressing comorbidities $(21,22)$.

\section{Hypertension}

Uncontrolled hypertension along with poor management of other co-morbid conditions has been considered responsible for re-hospitalizations in patients with HFpEF (22). Blood pressure (BP) control on its own is considered one of the most important preventive measures for HFpEF (19). Hypertension is the main cause of HFpEF with a $60-89 \%$ prevalence in large controlled trials, epidemiological studies and HF registries (4). The SPRINT research group showed that strict (BP) control (systolic BP $<120 \mathrm{mmHg}$ ) is associated with not only a significantly lower incidence of $\mathrm{HF}$, but also with a decrease in cardiovascular mortality. However, the trial did not differentiate between the incidence of HFpEF vs. HFrEF (22). In addition, the 2017 ACC/ AHA HF guidelines and the American Heart Failure and High Blood Pressure guidelines, recommend a lower systolic BP target of $<130 / 80 \mathrm{mmHg}(19,20)$. Patients with stage C HFpEF and hypertension should be treated with angiotensin converting enzyme inhibitors (ACEIs), angiotensin receptor blockers (ARBs), and beta blockers after achieving euvolemia with diuretics (class II a recommendation) $(4,19,20)$. At the same time, ARBs and aldosterone antagonists should be considered for reducing hospitalizations in HFpEF (class II b, level of evidence B) (19). The choice of BP medications should be tailored to co-existing conditions, tolerance and response. Vasodilators should be used with caution as they can cause undesirable side effects in patients with HFpEF (21).

The ALLHAT trial studied 42,418 high-risk hypertensive patients randomized to chlorthalidone, amlodipine, lisinopril, or doxazosin and compared the effect of these treatments in the number of hospitalizations for HFpEF or HFrEF. Results of this trial showed that chlorthalidone reduced the risk of HFpEF compared with amlodipine, lisinopril, or doxazosin (Table 1). Overall, it is important to acknowledge that HFpEF has a better prognosis than HFrEF in hypertensive men and women (23). In addition, given the causal relationship between obstructive sleep apnea and hypertension, formal sleep assessment should be considered in patients who meet clinical criteria for OSA $(19,24)$.

\section{CAD}

Regarding pharmacotherapy in patients with CAD, the 
Table 1 Medications with proven benefits in patients with heart failure with preserved ejection fraction

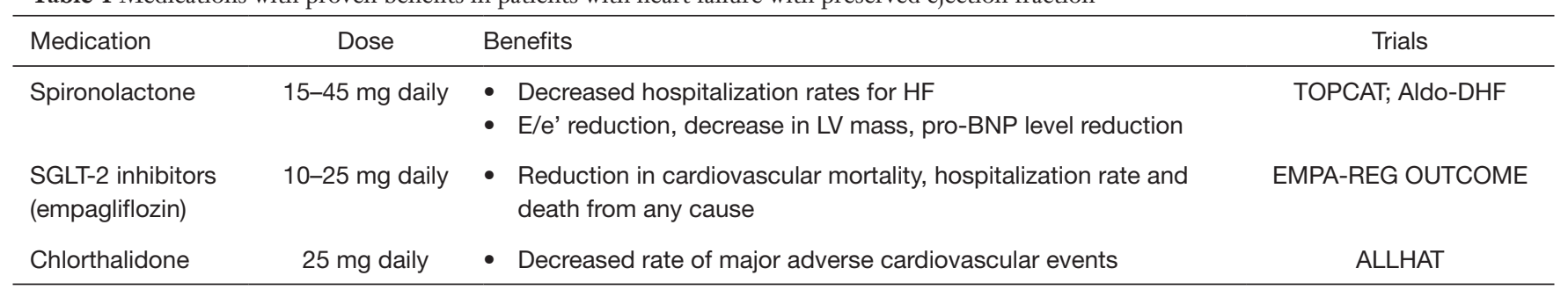

emphasis is to continue with guideline directed therapy. The question that will always arise is what role does revascularization play in these patients. According to the AHA/ACC 2017 guidelines, coronary revascularization is recommended in those patients whose symptoms (angina) or demonstrable myocardial ischemia adversely affect HFpEF despite medical therapy (class II a, Level C) $(4,21)$. An observational study of $376 \mathrm{HFpEF}$ patients $(68 \%$ with angiographically-proven CAD) showed that complete revascularization was associated with less decline in EF and lower mortality compared to those who were not completely revascularized, independent of other predictors [hazard ratio: $0.56 ; 95 \%$ confidence interval (CI), 0.33 to 0.93 ; $\mathrm{P}=0.03]$ (25). At the same time, other retrospective studies suggest that clinically evident acute coronary ischemia may not be the cause for acute decompensation in HFpEF. If there is no decrease in EF during an acute ischemic episode, then epicardial coronary revascularization may have at most a minor role in preventing a HFpEF recurrence (19). Overall, prospective studies are needed to determine if there is an optimal medical management strategy of CAD in HFpEF patients.

\section{Atrial fibrillation (AF)}

$\mathrm{AF}$ is common and can occur before, during or after the onset of HFpEF symptoms (21). AF contributes to HFpEF pathogenesis by exacerbating neuro-humoral activation as well as leading to a large, poorly contractile left atrium and impaired systolic and diastolic function of the left ventricle (LV) (19). In particular, tachycardia shortens the time of diastole and impairs adequate diastolic filling, with possible compromise of ventricular filling. These changes affect cardiac functional reserve and exercise tolerance. It is unknown what the best strategy is for AF management in patients with $\mathrm{HFpEF}$ as no trials have studied rate $v$. rhythm control in this population $(4,19)$. AF should be managed according to current guidelines and there is not data to support one rate controlling medication over another (26). A trial of rhythm control can be considered if symptoms persist despite adequate rate control. Rhythm control may be more beneficial when the HF presentation or exacerbation is associated with the onset of AF (21). Cardioversion is recommended to restore rhythm, and catheter ablation has limited long term benefits in HFpEF (19).

\section{Hyperlipidemia}

It has been suggested that statins can have potential benefits in diastolic HF independent of lipid control. These include a protective effect on LV hypertrophy, fibrosis, remodeling, mild antihypertensive, antioxidant and anti-inflammatory properties, and an increase in arterial elasticity with an effect on endothelial function and regression of aortic atherosclerosis (27). Observational studies have shown improved survival in patients with HFpEF on statin treatment, but it is questionable if there is a causal relationship $(21,28)$. Alehagen et al. studied 3,427 patients with $\mathrm{HF}$ ( $\mathrm{EF} \geq 50 \%$, age $77 \pm 11$ years and $54.0 \%$ women) treated with statins from the prospective Swedish Heart Failure Registry. The results of this study showed that statins were associated with reduced cardiovascular death (hazard ratio, 0.86; 95\% CI, 0.75-0.98; $\mathrm{P}=0.026$ ) and composite all-cause mortality or cardiovascular hospitalization (hazard ratio, 0.89; 95\% CI, 0.82-0.96; $\mathrm{P}=0.003$ ) (28). In addition, another observational single site study examined an association of statin therapy with an increase in two-year survival in 137 patients with diastolic HF $(27,29)$. Although treatment with ACE inhibitors, ARBs, beta blockers or calcium channel blockers had no significant effect on survival, treatment with statin was associated with survival benefit after adjustment for potential confounders (RR $0.20 ; 95 \%$ CI, 0.06 to $0.62 ; \mathrm{P}=0.005$ ). Of note after statin therapy, LDL cholesterol levels fell to a similar level as in patients not receiving a statin, $101 \pm 32$ vs. 
$98 \pm 33 \mathrm{mg} / \mathrm{dL}$ (27). The study was limited by a small sample size and could not determine if the benefit of statin therapy was independent of cholesterol levels (29).

Given that patients with HFpEF have co-morbidities, such as CAD, diabetes and impaired renal function, the benefit of statins in diastolic HF could be related to improved outcomes for these conditions (27). For this reason, the ACC/AHA guidelines do not recommended statins for the treatment of HFpEF alone, in the absence of other indications for their use (22).

\section{Obesity}

Tissue level inflammation associated with metabolic syndrome contributes to the development of HFpEF (29). Increased adiposity promotes inflammation, hypertension, insulin resistance and dyslipidemia, as well as impairing muscular metabolism and cardiovascular function contributing to exercise intolerance $(19,21,30)$. However, there is no role for a specific pharmacotherapeutic option, such as a weight loss agent. However, much like in those patients with $\mathrm{CAD}$, they should continue with routine care and therapy, and additional interventions can play a role in altering the disease severity. HFpEF is common in the elderly population and exercise intolerance is a predominant symptom that significantly impairs quality of life (QOL). Kitzman et al. developed the first randomized trial to test whether diet or exercise improved exercise capacity and QOL in older obese patients with chronic stable HFpEF $(19,30)$. Results of the study showed that aerobic exercise and diet increased peak oxygen consumption (peak $\mathrm{VO}_{2}$ in $\mathrm{mL} / \mathrm{kg} / \mathrm{min}$ ) and the combination of both interventions had an additive effect. Still, these interventions did not have a significant effect on the QOL measured by the Minnesota Living with Heart Failure Questionnaire. However, a metaanalysis of five randomized controlled trials showed that exercise training (ET) improved peak $\mathrm{VO}_{2}$, six-minute walk distance and QOL in patients with HFpEF in comparison to usual care $(31,32)$.

ET does not only improve functional capacity but can also prevent atrial and ventricular remodeling. In the EXDHF pilot trial, 64 patients with $\mathrm{HFpEF}$ were randomized to supervised endurance/resistance training and usual care vs. usual care alone. Peak $\mathrm{VO}_{2}$ increased with $\mathrm{ET}$ and E/e' (as a surrogate for LV filling pressures) and left atrial volume index decreased in comparison to the control group. These changes are associated with atrial reverse remodeling and improved left ventricular diastolic function $(20,22,30,33)$.
The long-term impact of ET on patient-related outcomes in HFpEF is still unclear. None of the studies published so far have been designed to address hospitalizations and mortality in these patients.

\section{Diabetes mellitus (DM) type 2}

Approximately $45 \%$ of HFpEF patients have DM, which is a predictor of increased morbidity and mortality in this population. This is illustrated in several large clinical trials that evaluated the characteristics of patients with $\mathrm{HFpEF}$ and DM and the role this disease plays as a prognostic factor. Increased cardiovascular death, HF hospitalizations and all-cause mortality was observed in CHARM, DIG and I-PRESERVE trials in patients with HFpEF and DM. These patients, in comparison to those without DM, were younger, had higher BMI, higher rates of ischemic heart disease, hypertension, lower exercise capacity and hypervolemia. Moreover, a retrospective cohort study of 232,656 subjects with HFpEF from the GWTG-HF registry, showed a significant increase in in-hospital and post discharge morbidity associated with DM (34).

Various DM medications including metformin, pioglitazone, incretins and SGLT2 inhibitors, have promising results in diabetic and non-diabetic patients with HFpEF. Observational studies have reported that metformin was associated with a $20 \%$ lower mortality, compared mostly with sulfonylureas (HR $0.80 ; 95 \%$ CI, 0.74-0.87; $\mathrm{P}<0.001)$ and a small reduction in all-cause hospitalizations (pooled adjusted risk estimate: 0.93; 95\% CI, 0.89-0.98; $\mathrm{P}=0.01$ ) in patients with HF (35). In particular, exenatide has been shown to improve diastolic function in patients with DM, as well as linagliptin and sitagliptin which showed similar results in this patient population with chronic kidney disease. The EMPAREG OUTCOME trial investigated the effects of empagliflozin (SGLT2 inhibitor) in patients with DM and found a relative risk reduction in cardiovascular mortality, hospitalization for heart failure, and death from any cause (Table 1). Empagliflozin is recommended in diabetic heart failure patients by the ESC guidelines in combination with metformin (IIa recommendation) (22). Additionally, the ongoing EMPEROR- Preserved Trial will study 5,750 HFpEF patients (EF $>40 \%$ ) with and without DM. Patients will be randomized to placebo or empagliflozin in addition to guideline-directed medical therapy. The primary end point is the time-to-first-event analysis of the combined risk for cardiovascular death or hospitalization for HF in a time 
frame of up to 38 months $(20,22,36)$. Other ongoing trials with SGLT2 inhibitors include EMBRACE-HF trial which investigates the change in pulmonary artery $(\mathrm{PA})$ diastolic pressure with empagliflozin and the PRESERVED-HF trial which evaluates the change in NT-pro BNP levels with dapagliflozin in patients with HFpEF and DM. The protective cardiovascular effect associated with this group of medications can be explained by the inhibition of sodiumglucose cotransporter 2, which reduces intravascular volume by promoting glucosuria, diuresis and natriuresis without sympathetic activation $(20,34)$.

Additionally, the TOPLEVEL trial is an ongoing randomized multicenter clinical trial that investigates whether DPP-IV inhibitor, Teneligliptin, can improve LV diastolic dysfunction in patients with DM. The primary endpoint measure is the change in $\mathrm{E} / \mathrm{e}$ ' by echocardiography from baseline and two years after enrollment $(20,37)$.

It is important to determine if anti-diabetic medications have an impact on cardiovascular outcomes in HFpEF and to understand the "phenotype" of this particular group of patients in order to find new therapeutic targets.

\section{Mineralocorticoid receptor antagonists: the role of spironolactone}

Aldosterone is involved in the pathogenesis of HFpEF and mineralocorticoid receptor antagonists (MRA) are a promising therapeutic alternative to improve $\mathrm{LV}$ diastolic dysfunction and remodeling (4,20). Vascular and cardiac mineralocorticoid receptors are stimulated by aldosterone to induce fibrosis, hypertrophy and endothelial dysfunction. In particular, the renin-angiotensin-aldosterone system (RAAS) promotes collagen deposition in the extracellular matrix, stimulates cardiac fibroblasts and induces the expression of profibrotic agents in an environment of inflammation and oxidative stress (20). In turn, these effects promote cardiovascular remodeling and increase left ventricular diastolic stiffness (20,31).

Given the beneficial effects of MRA on LV diastolic function, clinical studies have focused on determining if this group of medications improve outcomes in HFpEF. The TOPCAT is a randomized, double blind controlled trial that investigated 3,445 patients with symptomatic HF and a LVEF of $45 \%$ or more and assigned to either spironolactone (15-45 mg daily) or placebo (Table 1) (14). Subjects had a history of a HF hospitalization within the previous 12 months and elevated pro-BNP within 60 days before randomization. The median age was 69 years, $52 \%$ were female and the majority were NYHA class II $(20,29)$. Subjects had a mean follow-up of 3.3 years. The primary composite outcome was death from cardiovascular causes, aborted cardiac arrest or hospitalization for treatment of HF. Results showed that treatment with spironolactone did not significantly reduce the primary composite outcome (HR 0.89; 95\% CI, 0.77-1.04, $\mathrm{P}=0.14$ ). When each component of the primary composite was analyzed separately, spironolactone significantly decreased the hospitalization rate for HF in comparison to placebo by $17 \%$ (HR 0.83; 95\% CI, 0.69-0.99, $\mathrm{P}=0.04$ ) but neither total deaths nor hospitalizations for any other reason were significantly reduced in the spironolactone group $(4,14,20,21)$. It is important to note that treatment was associated with increased serum creatinine and double the rate of hyperkalemia $(14,21)$. However, there were no significant differences in the incidence of serious adverse events with frequent monitoring (14).

Heterogeneity between geographic regions was an important study limitation of the TOPCAT trial (4). Post hoc analyses showed spironolactone efficacy in HFpEF patients on primary outcome and on its two individual components (cardiovascular death and HF hospitalization) only in the Americas (HR 0.83) but not in Russia (HR 1.10). This led to question trial results in Russia and led to the belief that these may not reflect the true therapeutic response to spironolactone $(20,22)$. Based on this analysis, spironolactone may be beneficial in a selective population with symptomatic HFpEF particularly those with elevated BNP levels and it requires close monitoring of potassium levels and renal function (4).

Moreover, Aldo-DHF was a smaller randomized controlled trial that investigated 442 ambulatory patients with NYHA class II-III symptoms, echocardiographic evidence of diastolic dysfunction (grade $\geq \mathrm{I}$ ) and preserved EF (LV EF $>50 \%)$. Patients were randomized to spironolactone (25 $\mathrm{mg}$ daily) and placebo groups (22). The study showed significant improvements in diastolic LV function such as E/e' reduction, decrease in left ventricular mass (reverse remodeling) and reduction of the NP-pro BNP level, but no effects on exercise capacity or QOL $(20,22,38)$. There was a modest increase in serum potassium levels and a reduction in estimated glomerular filtration rate without an increase in hospitalization rate (38). The study showed spironolactone improved LV remodeling and neuro-hormonal activation (19).

The effect of exercise capacity with spironolactone was studied by Kosmala et al. in 150 patients with HFpEF. 
Subjects had exertional dyspnea (NYHA class II/III), echocardiographic evidence of diastolic dysfunction and baseline increased exercise E/e' ratio levels. Patients were randomized to spironolactone ( $25 \mathrm{mg}$ daily) or placebo for six months. The spironolactone group showed improvement in exercise capacity evidenced as an increment in peak oxygen consumption with reduction in exertional E/e' ratio $(20,39)$.

There is a role of aldosterone receptor antagonists in a subset of patients with HFpEF per the 2017 ACC/ AHA/HFSA guidelines based on the results of the clinical studies showing the benefits of spironolactone on diastolic dysfunction, predominantly the TOPCAT trial. Aldosterone receptor antagonists can be considered in selected HFpEF patients with $\mathrm{EF}>45 \%$, elevated BNP levels, HF admission within one year, estimated GFR $>30 \mathrm{~mL} / \mathrm{min}$, creatinine $<2.5 \mathrm{mg} / \mathrm{dL}$ and potassium $<5 \mathrm{mEq} / \mathrm{L}$, in order to decrease the number of hospitalizations (Class IIb, LOE B-R) $(4,20)$.

Finally, the SPIRRIT-HF is an ongoing phase III trial developed by the German Centre for Cardiovascular Research, to investigate the effects of spironolactone in 3,500 patients with HFpEF from the Swedish Heart Failure Registry. Subjects have EF $>40 \%$ and elevated pro-BNP levels (22). The primary outcomes is time to death from any cause during a five-year period ending by 2021 (20). Ongoing trials will hopefully provide an insight into the patient characteristics with HFpEF that will most likely benefit from MRA.

\section{Diuretics}

According to the ACC and ESC guidelines, diuretics are the only recommended intervention proven to relieve symptoms in hypervolemic patients with HFpEF (class IC/ IB respectively) $(4,20)$. These should be used at the lowest effective dose and adjusted according to the patient's body weight, symptoms and electrolyte status (21). Caution should be taken with excessive preload reduction induced by diuretics which can lead to LV under-filling and concomitant reduction of stroke volume and cardiac output, particularly in a hypertrophied LV. In the Hong Kong Diastolic HF Study, diuretic therapy significantly improved symptoms and, in combination with RAAS inhibitors, improved LV systolic and diastolic function, as well as lowered pro-BNP levels over a one-year period (22).

Besides symptomatic relief, diuretics may also improve outcomes when therapy is titrated based on central hemodynamic measurements as evidenced in the

\section{CHAMPION trial.}

The CHAMPION trial prospectively studied HF management based on frequent and remotely obtained PA pressures using CARDIOMEMs, a wireless PA pressure monitor implanted during a right heart catheterization. A total of 550 patients with HF associated with both preserved and reduced systolic function were included in the study. Subjects included 119 patients $(22 \%)$ with preserved $\mathrm{EF}$ (defined as $\mathrm{EF} \geq 40 \%$; average $51 \%$ ) and 66 patients with $\mathrm{LVEF} \geq 50 \%$. After implantation, the patients were randomly assigned to a treatment group that received therapy tailored to the hemodynamic data received by the implanted sensor in addition to standard HF management. The primary efficacy end point was rate of HF hospitalizations and it was $46 \%$ lower in the preserved EF treatment group compared to the control group at six months (incidence rate ratio, 0.54 ; 95\% CI, 0.38-0.70; $\mathrm{P}<0.0001)$ and $50 \%$ lower after an average 17.6 months follow-up (incidence rate ratio, 0.50; 95\% CI, 0.35-0.70; $\mathrm{P}<0.0001)(40)$.

Additional analysis revealed that hospitalization reductions were similar between patients in the subgroup with $\mathrm{LVEF} \geq 40 \%$ and the additional analysis performed in the group with LVEF $\geq 50 \%$. Results were consistent with previous hemodynamic monitoring trials that demonstrated an association between high filling pressures and increased hospitalization risk. The CHAMPION trial demonstrated that lowering PA pressures using diuretics, and additional vasodilators if pressures remained elevated, is an effective strategy to improve the outcome of patients with HFpEF. Results also suggest that the progressive elevation in filling pressures is a common mechanism leading to congestion in patients with HF independent of EF. Lowering these pressures with medical management maintains clinical stability and keeps patients out of the hospital for a significant follow-up period. Even though, a post hoc analysis of the patients with LVEF $\geq 50 \%$ in the CHAMPION trial showed similar outcomes to other groups, further studies are needed in this particular patient population (40).

\section{Heart rate reduction and sympathetic inhibition: beta blockers}

Heart failure induces many neurohormonal changes, including activation of the RAAS and the sympathetic system (41). By blocking adrenergic receptors, beta-blockers reduce heart rate, ventricular wall stress and the adrenergic 
stimulation, improving the neuro-endocrine response to HF (42-44). It has been shown in several trials and meta-analyses that in patients with HFrEF, beta-blockers reduce hospital admissions and decrease mortality (45-49). However, the indications to use beta-blockers in patients with HFpEF seem less clear due to conflicting data (50-52).

In a meta-analysis done by Liu et al., it was shown that the use of beta-blockers was associated with a statistically significant reduction in all-cause mortality but did not statistically improve the rate of hospitalization or the composite outcomes of mortality and hospitalization in patients with HFpEF. It is worth mentioning that the EF cutoff used was $\geq 40 \%$ (53).

In a more recent individual patient-level meta-analysis, beta-blockers were associated with a reduced risk of cardiovascular death in patients with an $\mathrm{EF}<50 \%$, and with a significantly reduced all-cause mortality in patients with $\mathrm{EF}<40 \%$. In the patient subgroup $(\mathrm{n}=244)$ with an EF $\geq 50 \%$ beta-blockers failed to show improvement in any of the prior outcomes (24).

In the absence of a strong alternate indication, the evidence to support the use of beta-blockers in patients with HFpEF is still lacking.

\section{Afterload reduction}

\section{ACEIs}

Continuous activation of the RAAS is associated with development of ventricular hypertrophy and myocardial fibrosis $(54,55)$. Inhibition of RAAS decreases neurohormone levels resulting in attenuation of $\mathrm{LV}$ hypertrophy and reducing morbidity and mortality in patients with HF (56). However, this last statement has not been proven in patients with $\mathrm{HFpEF}$.

The PEP-CHF trial assessed the efficacy of ACEIs, specifically perindopril in patients with HFpEF. The trial did not show a statistically significant benefit when compared with placebo on long-term morbidity and mortality. It is important to point that the trial had an inadequate power since many of the patients withdrew from assigned therapy to start open-label ACEIs. Treatment with perindopril did appear to improve symptoms and exercise capacity. It also showed that patients with prior myocardial infarction or with elevated systolic BP, who are at an increased risk of death or hospitalization, benefited from perindopril treatment during the first year follow-up (13). Ferrari previously had demonstrated that perindopril positively influences cardiac remodeling in patients who had post-infarction $\mathrm{HF}$ without $\mathrm{LV}$ systolic dysfunction (57).

Even though ACEIs have not been proven to reduce morbidity and mortality in patients with HFpEF, they do play an important role in the management of the comorbidities associated with this entity (e.g., hypertension and $\mathrm{CAD})$.

\section{Angiotensin II receptor blockers}

There is no evidence that ARBs decrease mortality in patients with HFpEF when compared to placebo. Two large randomized controlled trials, CHARM-preserved and I-PRESERVE, assessed the efficacy in HFpEF of placebo $v s$. candesartan and irbesartan, respectively. None of the trials have shown a difference in mortality when compared to placebo $(58,59)$. However, CHARM-preserved did show that patients treated with candesartan were less frequently hospitalized (58). A different trial demonstrated that ARBs are not superior to different types of antihypertensives to improve diastolic function (60). With the current evidence, ARBs seem to have the same important role as ACEIs in the treatment of comorbidities associated with HFpEF, but no direct benefit has been found.

\section{Angiotensin receptor/neprilysin inbibitor}

Release of atrial natriuretic peptide (ANP) and B-type natriuretic peptide (BNP) occurs as a response to cardiac myocyte stretch, which is secondary to increased myocardial wall tension. The release of these peptides acts as a defense mechanism to protect the heart from volume and pressure overload. This mechanism is impaired during the early stages of HFpEF (61).

The effects of ANP and BNP are not only natriuretic, vasodilatory and diuretic, but also inhibit the sympathetic nervous system, RAAS, release of antidiuretic hormone, improve myocardial relaxation and prevent myocyte hypertrophy and fibrosis $(62,63)$.

Neprilysin degrades ANP, BNP and C-type peptide, but not the inactive NT-proBNP (64). Neprilysin inhibitors, by increasing the levels of natriuretic peptides, stimulate diuresis, natriuresis, and vasodilation, which generates cyclic guanosine 3 '5' mono phosphate reducing hypertrophy and improving myocardial relaxation $(62,63)$. Neprilysin also causes breakdown of angiotensin, the reason for which dual-acting compounds were created, to inhibit neprilysin and block the action/generation of 
angiotensin (65).

Solomon et al. showed that administration of angiotensin receptor-neprilysin inhibitors (ARNi) reduced the levels of NT-proBNP significantly when compared to valsartan alone (66). Reductions of NTproBNP levels are associated with improved outcomes in patients with HF (67). The trial also demonstrated a significant reduction in the left atrial size in the group that received ARNi (66).

Until recently, ARNi have not shown improvement in morbidity and mortality in patients with preserved EF (68). However, a recent putative placebo analysis of the effects of sacubitril/valsartan showed that ARNi decrease the risk significantly of HF hospitalization and cardiovascular death across the full range of LVEF, with a more pronounced effect when $\mathrm{EF}$ is $<60 \%$ (69).

\section{Ineffective pharmacotherapy}

\section{Digoxin}

Digoxin has no effect in morbidity and mortality in patients with HFpEF and is not indicated to treat these patients, unless there is an associated condition that requires treatment with digitalis (70).

\section{Nitrates}

Evidence indicates that anomalies in the nitric oxide (NO)cyclic guanosine monophosphate (cGMP) pathway cause increased filling pressures and an inadequate cardiac output reserve in patients with HFpEF $(71,72)$. However, nitrates have not demonstrated effectiveness and are actually associated with worse outcomes $(73,74)$.

Inorganic sodium nitrite, administrated acutely during exercise, improves hemodynamic derangements, biventricular filling pressures and PA pressures, but administered chronically has failed to show improvement in exercise capacity (75-77).

\section{Phophodiesterase-5 inbibitors}

There is no improvement in exercise capacity, pulmonary artery wedge pressure (PAWP), cardiac output and peak oxygen consumption when phophodiesterase-5 inhibitors are administered $(78,79)$. Recent data suggests that they might play a role in prevention of HFpEF but further studies are needed (80).

\section{Guiding diuretic pharmacotherapy by implantable bemodynamic monitoring}

CardioMEMS HF System is an implantable device that measures and monitors heart rate and PA pressure providing information to guide outpatient HF medical management. The CHAMPION trial showed that patients with HFpEF whose treatment was guided by information provided by the device had less hospitalizations when compared to standard HF management (40). However, the efficacy of the device in this population is still under investigation (81).

\section{Conclusions}

In contrast to HFrEF, there are no treatment modalities that have shown to improve outcomes or survival in patients with HFpEF. Given the high co-morbidity burden and the impact these impose on mortality rates, HFpEF pharmacotherapy predominantly involves adequate recognition and disease classification followed by addressing the associated co-morbidities and symptom management. Currently, the benefit of medications including statins, ACEIs/ARBs and beta-blockers lies on the treatment for comorbidities but are not recommended solely for HFpEF. It is important to mention that mineralocorticoid receptor antagonists have shown improved outcomes in a subgroup of these patients. Similarly, pioglitazone, incretins and SGLT2 inhibitors have been shown to have a favorable effect on diastolic function, with benefits thought to be independent of a concomitant DM diagnosis. Diuretics continue to be the mainstay to provide symptomatic relief and have shown to reduce HF hospitalizations when therapy is tailored to central hemodynamic parameters. Understanding the HFpEF pathophysiology continues to be a matter of investigation which will be important in tailoring future pharmacologic strategies for this disease. Finally, regular physical activity and exercise improves exercise capacity and QOL in HFpEF and referral to a cardiac rehabilitation program including dynamic ET is reasonable in these individuals (82).

\section{Acknowledgments}

Funding: None.

\section{Footnote}

Provenance and Peer Review: This article was commissioned 
by the Guest Editors (Dr. Debabrata Mukherjee and Dr. Jose B. Cruz Rodriguez) for the series "Heart Failure Update and Advances in 2021" published in Annals of Translational Medicine. The article was sent for external peer review organized by the Guest Editors and the editorial office.

Reporting Checklist: The authors have completed the NARRATIVE REVIEW reporting checklist. Available at http://dx.doi.org/10.21037/atm-20-4602

Conflicts of Interest: All authors have completed the ICMJE uniform disclosure form (available at http://dx.doi. org/10.21037/atm-20-4602). The series "Heart Failure Update and Advances in 2021" was commissioned by the editorial office without any funding or sponsorship. DM served as the unpaid Guest Editor of the series. The authors have no other conflicts of interest to declare.

Ethical Statement: The authors are accountable for all aspects of the work in ensuring that questions related to the accuracy or integrity of any part of the work are appropriately investigated and resolved.

Open Access Statement: This is an Open Access article distributed in accordance with the Creative Commons Attribution-NonCommercial-NoDerivs 4.0 International License (CC BY-NC-ND 4.0), which permits the noncommercial replication and distribution of the article with the strict proviso that no changes or edits are made and the original work is properly cited (including links to both the formal publication through the relevant DOI and the license). See: https://creativecommons.org/licenses/by-nc-nd/4.0/.

\section{References}

1. Denolin H, Kuhn H, Krayenbuehl HP, et al. The definition of heart failure. Eur Heart J 1983;4:445-8.

2. Basuray A, French B, Ky B, et al. Heart failure with recovered ejection fraction: clinical description, biomarkers, and outcomes. Circulation 2014;129:2380-7.

3. Sweitzer NK, Lopatin M, Yancy CW, et al. Comparison of clinical features and outcomes of patients hospitalized with heart failure and normal ejection fraction ( $>$ or $=55 \%$ ) versus those with mildly reduced ( $40 \%$ to $55 \%$ ) and moderately to severely reduced ( $<40 \%$ ) fractions. Am J Cardiol 2008;101:1151-6.

4. Yancy CW, Jessup M, Bozkurt B, et al. 2013 ACCF/AHA guideline for the management of heart failure: a report of the American College of Cardiology Foundation/American Heart Association Task Force on Practice Guidelines. J Am Coll Cardiol 2013;62:e147-239.

5. Levy D, Kenchaiah S, Larson MG, et al. Long-term trends in the incidence of and survival with heart failure. $\mathrm{N}$ Engl J Med 2002;347:1397-402.

6. Owan TE, Hodge DO, Herges RM, et al. Trends in prevalence and outcome of heart failure with preserved ejection fraction. N Engl J Med 2006;355:251-9.

7. Roger VL, Weston SA, Redfield MM, et al. Trends in heart failure incidence and survival in a community-based population. JAMA 2004;292:344-50.

8. Steinberg BA, Zhao X, Heidenreich PA, et al. Trends in patients hospitalized with heart failure and preserved left ventricular ejection fraction: prevalence, therapies, and outcomes. Circulation 2012;126:65-75.

9. Fonarow GC, Stough WG, Abraham WT, et al. Characteristics, treatments, and outcomes of patients with preserved systolic function hospitalized for heart failure: a report from the OPTIMIZE-HF Registry. J Am Coll Cardiol 2007;50:768-77.

10. Yancy CW, Lopatin M, Stevenson LW, et al. Clinical presentation, management, and in-hospital outcomes of patients admitted with acute decompensated heart failure with preserved systolic function: a report from the Acute Decompensated Heart Failure National Registry (ADHERE) Database. J Am Coll Cardiol 2006;47:76-84.

11. Dunlay SM, Roger VL, Redfield MM. Epidemiology of heart failure with preserved ejection fraction. Nat Rev Cardiol 2017;14:591-602.

12. Somaratne JB, Berry C, McMurray JJ, et al. The prognostic significance of heart failure with preserved left ventricular ejection fraction: a literature-based metaanalysis. Eur J Heart Fail 2009;11:855-62.

13. Cleland JG, Tendera M, Adamus J, et al. The perindopril in elderly people with chronic heart failure (PEP-CHF) study. Eur Heart J 2006;27:2338-45.

14. Pitt B, Pfeffer MA, Assmann SF, et al. Spironolactone for heart failure with preserved ejection fraction. $\mathrm{N}$ Engl J Med 2014;370:1383-92.

15. Lee DS, Gona P, Vasan RS, et al. Relation of disease pathogenesis and risk factors to heart failure with preserved or reduced ejection fraction: insights from the framingham heart study of the national heart, lung, and blood institute. Circulation 2009;119:3070-7.

16. Tribouilloy C, Rusinaru D, Mahjoub H, et al. Prognosis of heart failure with preserved ejection fraction: a 5 
year prospective population-based study. Eur Heart J 2008;29:339-47.

17. Anjan VY, Loftus TM, Burke MA, et al. Prevalence, clinical phenotype, and outcomes associated with normal B-type natriuretic peptide levels in heart failure with preserved ejection fraction. Am J Cardiol 2012;110:870-6.

18. Paulus WJ, Tschope C. A novel paradigm for heart failure with preserved ejection fraction: comorbidities drive myocardial dysfunction and remodeling through coronary microvascular endothelial inflammation. J Am Coll Cardiol 2013;62:263-71.

19. Upadhya B, Kitzman DW. Heart failure with preserved ejection fraction: New approaches to diagnosis and management. Clin Cardiol 2020;43:145-55.

20. Iliesiu AM, Hodorogea AS. Treatment of Heart Failure with Preserved Ejection Fraction. Adv Exp Med Biol 2018;1067:67-87.

21. Redfield MM. Heart Failure with Preserved Ejection Fraction. N Engl J Med 2016;375:1868-77.

22. Tschope C, Birner C, Bohm M, et al. Heart failure with preserved ejection fraction: current management and future strategies : Expert opinion on the behalf of the Nucleus of the "Heart Failure Working Group" of the German Society of Cardiology (DKG). Clin Res Cardiol 2018;107:1-19.

23. Davis BR, Kostis JB, Simpson LM, et al. Heart failure with preserved and reduced left ventricular ejection fraction in the antihypertensive and lipid-lowering treatment to prevent heart attack trial. Circulation 2008;118:2259-67.

24. Cleland JGF, Bunting KV, Flather MD, et al. Betablockers for heart failure with reduced, mid-range, and preserved ejection fraction: an individual patient-level analysis of double-blind randomized trials. Eur Heart J 2018;39:26-35.

25. Hwang SJ, Melenovsky V, Borlaug BA. Implications of coronary artery disease in heart failure with preserved ejection fraction. J Am Coll Cardiol 2014;63:2817-27.

26. Aurigemma GP, Gasch WH. Clinical practice. Diastolic heart failure. N Engl J Med 2004;351:1097-105.

27. Fukuta H, Sane DC, Brucks S, et al. Statin therapy may be associated with lower mortality in patients with diastolic heart failure: a preliminary report. Circulation 2005;112:357-63.

28. Alehagen U, Benson L, Edner M, et al. Association Between Use of Statins and Mortality in Patients With Heart Failure and Ejection Fraction of $>/=50$. Circ Heart Fail 2015;8:862-70.

29. Oren O, Goldberg S. Heart Failure with Preserved
Ejection Fraction: Diagnosis and Management. Am J Med 2017;130:510-6.

30. Kitzman DW, Brubaker P, Morgan T, et al. Effect of Caloric Restriction or Aerobic Exercise Training on Peak Oxygen Consumption and Quality of Life in Obese Older Patients With Heart Failure With Preserved Ejection Fraction: A Randomized Clinical Trial. JAMA 2016;315:36-46.

31. Gazewood JD, Turner PL. Heart Failure with Preserved Ejection Fraction: Diagnosis and Management. Am Fam Physician 2017;96:582-8.

32. Fukuta H, Goto T, Wakami K, et al. Effects of drug and exercise intervention on functional capacity and quality of life in heart failure with preserved ejection fraction: A meta-analysis of randomized controlled trials. Eur J Prev Cardiol 2016;23:78-85.

33. Edelmann F, Gelbrich G, Dungen HD, et al. Exercise training improves exercise capacity and diastolic function in patients with heart failure with preserved ejection fraction: results of the Ex-DHF (Exercise training in Diastolic Heart Failure) pilot study. J Am Coll Cardiol 2011;58:1780-91.

34. McHugh K, DeVore AD, Wu J, et al. Heart Failure With Preserved Ejection Fraction and Diabetes: JACC State-ofthe-Art Review. J Am Coll Cardiol 2019;73:602-11.

35. Eurich DT, Weir DL, Majumdar SR, et al. Comparative safety and effectiveness of metformin in patients with diabetes mellitus and heart failure: systematic review of observational studies involving 34,000 patients. Circ Heart Fail 2013;6:395-402.

36. Anker SD, Butler J, Filippatos GS, et al. Evaluation of the effects of sodium-glucose co-transporter 2 inhibition with empagliflozin on morbidity and mortality in patients with chronic heart failure and a preserved ejection fraction: rationale for and design of the EMPEROR-Preserved Trial. Eur J Heart Fail 2019;21:1279-87.

37. Imazu M, Nakano A, Ito $S$, et al. Effects of Teneligliptin on the Progressive Left Ventricular Diastolic Dysfunction in Patients with Type 2 Diabetes Mellitus in OpenLabel, Marker-Stratified Randomized, Parallel-Group Comparison, Standard Treatment-Controlled Multicenter Trial (TOPLEVEL Study): Rationale and Study Design. Cardiovasc Drugs Ther 2019;33:363-70.

38. Edelmann F, Wachter R, Schmidt AG, et al. Effect of spironolactone on diastolic function and exercise capacity in patients with heart failure with preserved ejection fraction: the Aldo-DHF randomized controlled trial. JAMA 2013;309:781-91. 
39. Kosmala W, Rojek A, Przewlocka-Kosmala M, et al. Effect of Aldosterone Antagonism on Exercise Tolerance in Heart Failure With Preserved Ejection Fraction. J Am Coll Cardiol 2016;68:1823-34.

40. Adamson PB, Abraham WT, Bourge RC, et al. Wireless pulmonary artery pressure monitoring guides management to reduce decompensation in heart failure with preserved ejection fraction. Circ Heart Fail 2014;7:935-44.

41. Remme WJ, Swedberg K. Comprehensive guidelines for the diagnosis and treatment of chronic heart failure. Task force for the diagnosis and treatment of chronic heart failure of the European Society of Cardiology. Eur J Heart Fail 2002;4:11-22.

42. von Lueder TG, Kotecha D, Atar D, et al. Neurohormonal Blockade in Heart Failure. Card Fail Rev 2017;3:19-24.

43. Lopez-Sendon J, Swedberg K, McMurray J, et al. Expert consensus document on beta-adrenergic receptor blockers. Eur Heart J 2004;25:1341-62.

44. Hall SA, Cigarroa CG, Marcoux L, et al. Time course of improvement in left ventricular function, mass and geometry in patients with congestive heart failure treated with beta-adrenergic blockade. J Am Coll Cardiol 1995;25:1154-61.

45. Effect of carvedilol on mortality and morbidity in patients with chronic heart failure. Circulation 1996;94:592.

46. The Cardiac Insufficiency Bisoprolol Study II (CIBIS-II): a randomised trial. Lancet 1999;353:9-13.

47. Effect of metoprolol CR/XL in chronic heart failure: Metoprolol CR/XL Randomised Intervention Trial in Congestive Heart Failure (MERIT-HF). Lancet 1999;353:2001-7.

48. Packer M, Coats AJ, Fowler MB, et al. Effect of carvedilol on survival in severe chronic heart failure. $\mathrm{N}$ Engl J Med 2001;344:1651-8.

49. Shibata MC, Flather MD, Wang D. Systematic review of the impact of beta blockers on mortality and hospital admissions in heart failure. Eur J Heart Fail 2001;3:351-7.

50. Yamamoto K, Origasa H, Hori M. Effects of carvedilol on heart failure with preserved ejection fraction: the Japanese Diastolic Heart Failure Study (J-DHF). Eur J Heart Fail 2013;15:110-8.

51. Flather MD, Shibata MC, Coats AJ, et al. Randomized trial to determine the effect of nebivolol on mortality and cardiovascular hospital admission in elderly patients with heart failure (SENIORS). Eur Heart J 2005;26:215-25.

52. Conraads VM, Metra M, Kamp O, et al. Effects of the long-term administration of nebivolol on the clinical symptoms, exercise capacity, and left ventricular function of patients with diastolic dysfunction: results of the ELANDD study. Eur J Heart Fail 2012;14:219-25.

53. Liu F, Chen Y, Feng X, et al. Effects of beta-blockers on heart failure with preserved ejection fraction: a metaanalysis. PLoS One 2014;9:e90555.

54. McEwan PE, Gray GA, Sherry L, et al. Differential effects of angiotensin II on cardiac cell proliferation and intramyocardial perivascular fibrosis in vivo. Circulation 1998;98:2765-73.

55. Ramires FJ, Sun Y, Weber KT. Myocardial fibrosis associated with aldosterone or angiotensin II administration: attenuation by calcium channel blockade. J Mol Cell Cardiol 1998;30:475-83.

56. Benedict CR, Weiner DH, Johnstone DE, et al. Comparative neurohormonal responses in patients with preserved and impaired left ventricular ejection fraction: results of the Studies of Left Ventricular Dysfunction (SOLVD) Registry. The SOLVD Investigators. J Am Coll Cardiol 1993;22:146a-53a.

57. Ferrari R. Effects of angiotensin-converting enzyme inhibition with perindopril on left ventricular remodeling and clinical outcome: results of the randomized Perindopril and Remodeling in Elderly with Acute Myocardial Infarction (PREAMI) Study. Arch Intern Med 2006;166:659-66.

58. Yusuf S, Pfeffer MA, Swedberg K, et al. Effects of candesartan in patients with chronic heart failure and preserved left-ventricular ejection fraction: the CHARMPreserved Trial. Lancet 2003;362:777-81.

59. Massie BM, Carson PE, McMurray JJ, et al. Irbesartan in patients with heart failure and preserved ejection fraction. N Engl J Med 2008;359:2456-67.

60. Solomon SD, Janardhanan R, Verma A, et al. Effect of angiotensin receptor blockade and antihypertensive drugs on diastolic function in patients with hypertension and diastolic dysfunction: a randomised trial. Lancet 2007;369:2079-87.

61. Daniels LB, Maisel AS. Natriuretic peptides. J Am Coll Cardiol 2007;50:2357-68.

62. Potter LR, Abbey-Hosch S, Dickey DM. Natriuretic peptides, their receptors, and cyclic guanosine monophosphate-dependent signaling functions. Endocr Rev 2006;27:47-72.

63. Gardner DG, Chen S, Glenn DJ, et al. Molecular biology of the natriuretic peptide system: implications for physiology and hypertension. Hypertension 2007;49:419-26.

64. Martinez-Rumayor A, Richards AM, Burnett JC, et 
al. Biology of the natriuretic peptides. Am J Cardiol 2008;101:3-8.

65. Richards AM, Wittert GA, Crozier IG, et al. Chronic inhibition of endopeptidase 24.11 in essential hypertension: evidence for enhanced atrial natriuretic peptide and angiotensin II. J Hypertens 1993;11:407-16.

66. Solomon SD, Zile M, Pieske B, et al. The angiotensin receptor neprilysin inhibitor LCZ696 in heart failure with preserved ejection fraction: a phase 2 double-blind randomised controlled trial. Lancet 2012;380:1387-95.

67. Masson S, Latini R, Anand IS, et al. Prognostic value of changes in $\mathrm{N}$-terminal pro-brain natriuretic peptide in Val-HeFT (Valsartan Heart Failure Trial). J Am Coll Cardiol 2008;52:997-1003.

68. Solomon SD, McMurray JJV, Anand IS, et al. AngiotensinNeprilysin Inhibition in Heart Failure with Preserved Ejection Fraction. N Engl J Med 2019;381:1609-20.

69. Vaduganathan M, Jhund PS, Claggett BL, et al. A putative placebo analysis of the effects of sacubitril/valsartan in heart failure across the full range of ejection fraction. Eur Heart J 2020;41:2356-62.

70. Ahmed A, Rich MW, Fleg JL, et al. Effects of digoxin on morbidity and mortality in diastolic heart failure: the ancillary digitalis investigation group trial. Circulation 2006;114:397-403.

71. Borlaug BA, Olson TP, Lam CS, et al. Global cardiovascular reserve dysfunction in heart failure with preserved ejection fraction. J Am Coll Cardiol 2010;56:845-54.

72. van Heerebeek L, Hamdani N, Falcao-Pires I, et al. Low myocardial protein kinase $\mathrm{G}$ activity in heart failure with preserved ejection fraction. Circulation 2012;126:830-9.

73. Redfield MM, Anstrom KJ, Levine JA, et al. Isosorbide Mononitrate in Heart Failure with Preserved Ejection Fraction. N Engl J Med 2015;373:2314-24.

74. Lim SL, Benson L, Dahlstrom U, et al. Association Between Use of Long-Acting Nitrates and Outcomes in Heart Failure With Preserved Ejection Fraction. Circ

Cite this article as: Paz PA, Mantilla BD, Argueta EE, Mukherjee D. Narrative review: the holy grail: update on pharmacotherapy for heart failure with preserved ejection fraction. Ann Transl Med 2021;9(6):523. doi: 10.21037/atm-204602
Heart Fail 2017;10:e003534.

75. Borlaug BA, Melenovsky V, Koepp KE. Inhaled Sodium Nitrite Improves Rest and Exercise Hemodynamics in Heart Failure With Preserved Ejection Fraction. Circ Res 2016;119:880-6.

76. Borlaug BA, Koepp KE, Melenovsky V. Sodium Nitrite Improves Exercise Hemodynamics and Ventricular Performance in Heart Failure With Preserved Ejection Fraction. J Am Coll Cardiol 2015;66:1672-82.

77. Borlaug BA, Anstrom KJ, Lewis GD, et al. Effect of Inorganic Nitrite vs Placebo on Exercise Capacity Among Patients With Heart Failure With Preserved Ejection Fraction: The INDIE-HFpEF Randomized Clinical Trial. JAMA 2018;320:1764-73.

78. Redfield MM, Chen HH, Borlaug BA, et al. Effect of phosphodiesterase-5 inhibition on exercise capacity and clinical status in heart failure with preserved ejection fraction: a randomized clinical trial. JAMA 2013;309:1268-77.

79. Hoendermis ES, Liu LC, Hummel YM, et al. Effects of sildenafil on invasive haemodynamics and exercise capacity in heart failure patients with preserved ejection fraction and pulmonary hypertension: a randomized controlled trial. Eur Heart J 2015;36:2565-73.

80. Guazzi M, van Heerebeek L, Paulus WJ. Phosphodiesterase-5 inhibition in heart failure with preserved ejection fraction: trading therapy for prevention. Eur J Heart Fail 2017;19:337-9.

81. Loh JP, Barbash IM, Waksman R. Overview of the 2011 Food and Drug Administration Circulatory System Devices Panel of the Medical Devices Advisory Committee Meeting on the CardioMEMS Champion Heart Failure Monitoring System. J Am Coll Cardiol 2013;61:1571-6.

82. Pandey A, Parashar A, Kumbhani D, et al. Exercise training in patients with heart failure and preserved ejection fraction: meta-analysis of randomized control trials. Circ Heart Fail 2015;8:33-40. 